\title{
Review
}

\section{The Effects of Exercise on Dopamine Neurotransmission in Parkinson's Disease: Targeting Neuroplasticity to Modulate Basal Ganglia Circuitry}

\author{
G.M. Petzinger ${ }^{\mathrm{a}, \mathrm{c}, *}$, D.P. Holschneider ${ }^{\mathrm{a}, \mathrm{b}}$, B.E. Fisher ${ }^{\mathrm{a}, \mathrm{c}}$, S. McEwen ${ }^{\mathrm{a}}$, N. Kintz ${ }^{\mathrm{a}}$, M. Halliday ${ }^{\mathrm{a}}$, \\ W. Toy ${ }^{\mathrm{a}}$, J.W. Walsh ${ }^{\mathrm{d}}$, J. Beeler ${ }^{\mathrm{e}}$ and M.W. Jakowec ${ }^{\mathrm{a}, \mathrm{c}}$ \\ ${ }^{a}$ Department of Neurology, University of Southern California, Los Angeles, CA, USA \\ ${ }^{\mathrm{b}}$ Department of Psychiatry and the Behavioral Sciences, University of Southern California, Los Angeles, CA, USA \\ ${ }^{\mathrm{c}}$ Division of Biokinesiology and Physical Therapy, University of Southern California, Los Angeles, CA, USA \\ ${ }^{\mathrm{d}}$ Department of Psychiatry \& Biobehavioral Sciences, Andrus Gerontology, University of Southern California, \\ Los Angeles, CA, USA \\ ${ }^{\mathrm{e}}$ Department of Psychology, CUNY, NY, USA
}

\begin{abstract}
Animal studies have been instrumental in providing evidence for exercise-induced neuroplasticity of corticostriatal circuits that are profoundly affected in Parkinson's disease. Exercise has been implicated in modulating dopamine and glutamate neurotransmission, altering synaptogenesis, and increasing cerebral blood flow. In addition, recent evidence supports that the type of exercise may have regional effects on brain circuitry, with skilled exercise differentially affecting frontal-striatal related circuits to a greater degree than pure aerobic exercise. Neuroplasticity in models of dopamine depletion will be reviewed with a focus on the influence of exercise on the dorsal lateral striatum and prefrontal related circuitry underlying motor and cognitive impairment in PD. Although clearly more research is needed to address major gaps in our knowledge, we hypothesize that the potential effects of exercise on inducing neuroplasticity in a circuit specific manner may occur through synergistic mechanisms that include the coupling of an increasing neuronal metabolic demand and increased blood flow. Elucidation of these mechanisms may provide important new targets for facilitating brain repair and modifying the course of disease in PD.
\end{abstract}

Keywords: Synaptic plasticity, basal ganglia, prefrontal cortex, glutamate, cognition

\section{INTRODUCTION}

This manuscript presents an overview of the impact of exercise on neuroplasticity in animal models of Parkinson's disease (PD). Neuroplasticity is the ability of the brain to encode and learn new behaviors and can be defined as changes in molecular and cellular processes

\footnotetext{
*Correspondence to: Giselle M. Petzinger, MD, Department of Neurology, University of Southern California, Los Angeles, 90033 CA, USA. Tel.: +1 323442 1057; E-mail: gpetzinger@ surgery.usc.edu.
}

in response to environmental experiences such as exercise. We briefly explore the effects of exercise in the basal ganglia (called the striatum in rodents), pertinent neurotransmitter systems and associated cortical circuitry. While this brain area and related circuitry are known to be impaired in individuals with PD, exercise may help to restore the normal motor and cognitive function observed in healthy individuals, Exercise has been shown to affect a number of different neurotransmitters including dopamine [1, 2], glutamate [1, 3, 4], serotonin $[5,6]$, norepinephrine [6-8], and acetylcholine 
$[9,10]$ potentially contributing to the exercise related benefits observed in PD. This review will focus on two neurotransmitter systems that are essential for normal corticostriatal connectivity and function. Namely, exercise effects in dopamine (DA) and glutamate neurotransmission as well as neuronal connectivity (dendritic morphology) in basal ganglia circuits will be addressed. Additionally, while a wide variety of exercises have been reported to be beneficial in $\mathrm{PD}$, this review will also highlight recent animal studies that compare the type of exercise. By way of differential effects on blood flow and neurogenesis, skilled vs. aerobic exercise may each have a distinct impact on neuroplasticity. These differential effects which are brain region and circuit specific suggest a potential interaction between the type of exercise and its impact on induced neuronal activation and regional blood flow that may be important for facilitating repair or disease modification. Understanding the impact of exercise in the basal ganglia and its related circuitry may represent a new frontier in understanding mechanisms of neuroplasticity and repair and thus lead to novel therapeutic targets for PD.

\section{PARKINSON'S DISEASE AND EXERCISE AS A MODEL FOR NEUROPLASTICITY}

PD is a progressive neurodegenerative disorder that is characterized by the depletion of DA due to the degeneration of neurons in the substantia nigra pars compacta (SNpc), and to a lesser degree the ventral tegmental area (VTA). Characteristic features of PD include motor (bradykinesia, rigidity, tremor, gait dysfunction, and postural instability) and cognitive impairment (frontal lobe, executive dysfunction), as well as mood disorders. In PD, studies in exercise and

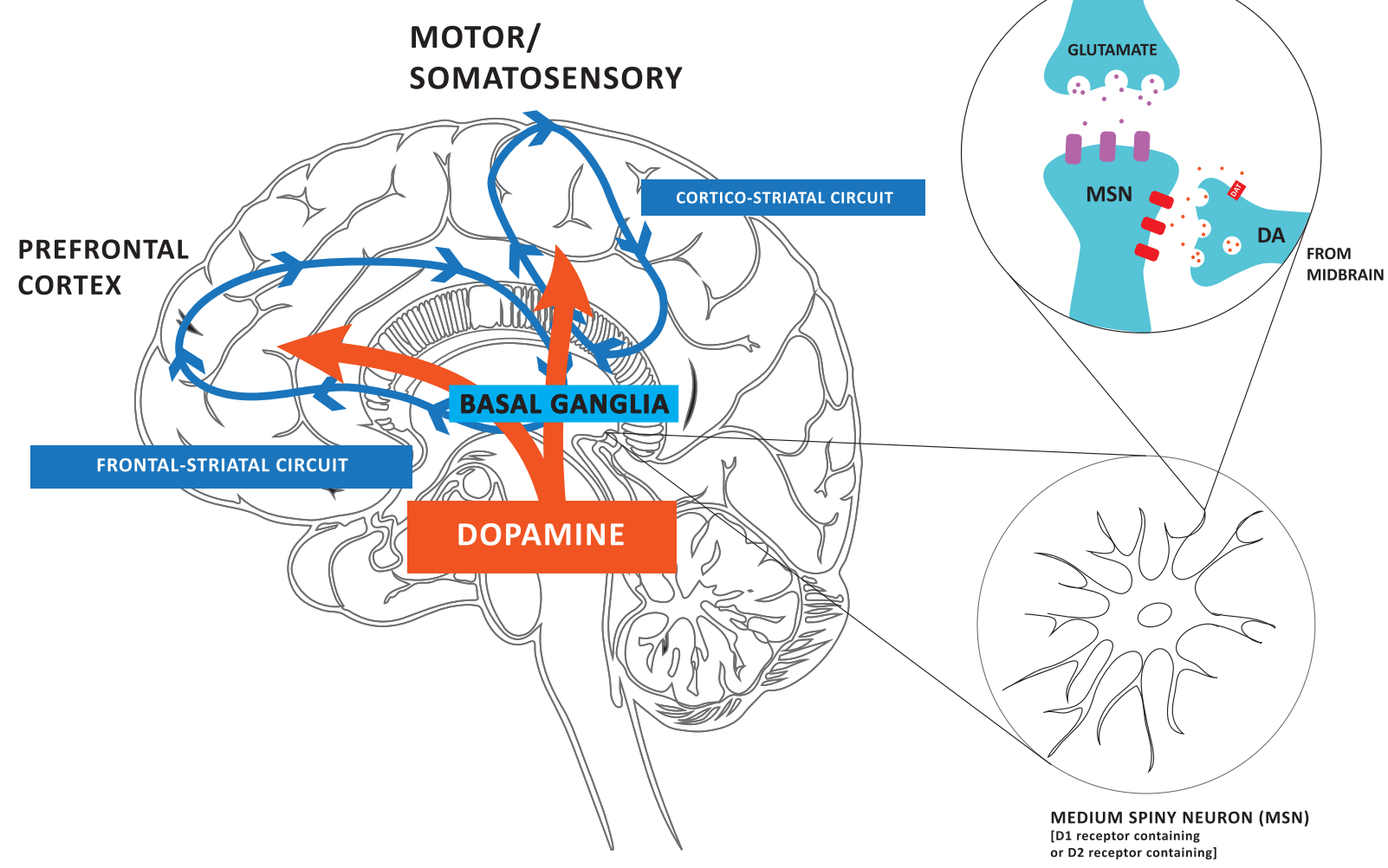

Fig. 1. Dopamine (DA) projections play a critical role in modulating both motor and cognitive circuits. Dopamine (DA) from neurons within the substantia nigra pars compacta and ventral tegmental area of the midbrain project to the dorsal lateral striatum of the basal ganglia and the prefrontal cortex, respectively. The earlier and more profound depletion of DA in the dorsal lateral striatum results in impairment in corticostriatal thalamic circuitry, which is important for automatic movements, and consequently greater reliance on frontal striatal circuitry, important for goal-directed motor control in Parkinson's disease (PD). Although affected to a lesser degree, DA loss in the frontal-striatal circuit contributes to cognitive impairments in PD. Animal studies are beginning to reveal evidence for exercise-induced neuroplasticity in motor and cognitive related circuitry in PD and how the two circuits are inter-related. 
neuroplasticity have focused on the basal ganglia and its cortical connections, since they comprise important motor and cognitive circuits, respectively, that are altered in disease. The basal ganglia consists of the putamen and caudate nucleus, collectively termed the striatum in rodents. The striatum is composed of DA- $\mathrm{D}_{1} \mathrm{R}$ and $\mathrm{DA}-\mathrm{D}_{2} \mathrm{R}$-containing medium spiny neurons (MSNs) of the direct and indirect projection pathways, respectively. Synaptic connections between DA-D ${ }_{1} \mathrm{R}$ and DA-D $\mathrm{D}_{2} \mathrm{R}$-containing MSNs and cortical glutamatergic neurons, make up cortical-striatal circuits [11]. In the healthy brain, these circuits are responsible for automatic (unconscious) and volitional (goal-directed) movements as well as cognitive processes, including executive function (EF) [12]. Executive function consists of working memory, task flexibility, and problem solving, as well as planning and execution of tasks [13]. The key circuits affected in PD are (i) the cortico-striatal motor circuit, including the dorsal lateral striatum (analogous to the putamen in primates), the primary motor and somatosensory cortex and the thalamus, and (ii) the frontal-striatal circuit, including the prefrontal cortex and the dorsal medial striatum (analogous to the caudate nucleus in primates). In Fig. 1 we depict the two major cortico-striatal circuits discussed in this review, their convergence in the striatum and modulation through DA. In PD the early and more profound DA-depletion occurs in the dorsal lateral striatum, thus leading to early deficits in automatic execution of routine movements [13, 14]. Imaging studies suggest that as individuals with PD lose control of automatic movements that there is a shift towards frontal-striatal volitional control of motor performance [15]. It has been posited that deficits in EF that are common even in early stages of PD may be due in part to an overrecruitment and saturation of the frontal-striatal circuit [16]. Alternatively, lesion studies have supported that direct impairment of the dorsal striatum may lead to disruption of the frontal striatal circuit and thus EF deficits directly [17]. In addition to their role in $\mathrm{PD}$, the two circuits described above as well as DA receptors are also important in motor learning [18]. Specifically, the volitional and automatic circuits and the DA- $D_{1} R$ and DA- $D_{2} R$ are involved in the acquisition phase of motor learning while the automatic circuit and the DA- $\mathrm{D}_{2} \mathrm{R}$ are involved in the retention phase of motor skill learning. Exercise that incorporates aspects of motor learning, such as skill (e.g., yoga, tai chi, treadmill running) may be useful for examining exercise-induced mechanisms of neuroplasticity in PD.

\section{EPIDEMIOLOGICAL STUDIES OF EXERCISE EFFECTS IN PD}

Physical activity has been demonstrated to lead to tremendous health benefits in individuals of all ages and in both healthy and disease states. It is only in the last two decades that epidemiological studies have suggested that a lifetime of physical activity may provide protection from a wide range of neurological disorders, including PD [19], Alzheimer's disease (AD) [20], and cognitive impairment associated with aging [21]. For example, a study by Chen and colleagues demonstrated that maintaining strenuous levels of physical activity in young adulthood was associated with a reduced risk of acquiring PD in later life [22]. One potential mechanism by which exercise may reduce an individual's risk for common neurodegenerative diseases, or age-related cognitive decline is through enhanced brain connectivity, with concomitant increased reserve and resilience to agerelated synaptic deterioration. These exercise-induced changes in brain connectivity may occur at a molecular and circuit level and include essential components that drive neuroplasticity: neurotransmission, synaptogenesis and neurogenesis. While, evidence suggests that impaired function in PD can be improved through rehabilitation and exercise, there remains a significant gap in understanding exercise-induced neuroplasticity in the context of a neurodegenerative disorder, such as PD. To elucidate the underlying mechanisms of exercise-related functional improvement in people with $\mathrm{PD}$, researchers have primarily utilized rodent models, including the neurotoxin induced 1methyl-4-phenyl-1, 2, 3, 6-tetrahydropyridine (MPTP) and 6-hydroxydopamine (6-OHDA) models [23, 24]. These models are helpful as a means to investigate exercise induced mechanisms of neuroplasticity and brain repair in PD since they exhibit analogous pathophysiological and behavioral characteristics of PD: (i) loss of midbrain dopaminergic neurons, (ii) depletion of striatal DA, (iii) aberrant corticostriatal connectivity and (iv) impaired cognitive and motor performance.

\section{EXERCISE EFFECTS ON DA NEUROTRANSMISSION IN ANIMAL MODELS}

In PD, the classical pathophysiological model is that the loss of DA in the dorsal lateral striatum leads to imbalance of the DA- $\mathrm{D}_{1} \mathrm{R}$ direct and DA-D $2 \mathrm{R}$ indirect pathways, such that there is increased 
and aberrant corticostriatal glutamatergic synaptic drive and hyper-excitability in the DA- $\mathrm{D}_{2} \mathrm{R}$ indirect containing pathway. It has been posited that restoration of DA neurotransmission along this DA- $\mathrm{D}_{2} \mathrm{R}$ pathway may serve to normalize this aberrant form of corticostriatal synaptic plasticity. Animal studies support that exercise benefits in PD may be due in part to facilitated DA neurotransmission. Specifically, using the MPTP mouse model of PD, intensive daily treadmill exercise leads to improved motor function and increased DA neurotransmission compared to the non-exercise MPTP mice. While both MPTP mice groups showed equal levels of cell loss and DA-depletion, only exercised mice showed: (i) increased evoked DA release, and (ii) increased extracellular DA though down regulation of the DA transporter (DAT) expression and (iii) decreased clearance using fast-scan cyclic voltammetry within the dorsal striatum $[1,2]$. In the context of motor learning and its potential role in exercise and rehabilitation related benefits in $\mathrm{PD}$, studies in mice report that DA availability can influence motor learning (rotarod training). Specifically, PitX3 (paired-like homeodomain transcription factor 3) mutant mice that lack striatal DA due to developmental loss of nigrostriatal dopaminergic neurons show deficiencies in motor learning [25]. Conversely, restoration of DA through levodopa treatment in these mutant mice restores motor learning [25]. Thus, exercise effects on DA availability through altered neurotransmission may act in part to promote mechanisms critical for motor learning and important for restoring motor behavior in PD.

Another mechanism by which exercise can influence DA neurotransmission is through DA receptor expression [26]. For example, exercise studies in rodents have demonstrated increased DA neurotransmission through an increase in DA- $\mathrm{D}_{2} \mathrm{R}$ protein expression and binding within the dorsal lateral striatum [1]. Specifically, after 28-days of intensive treadmill training in MPTP mice, $\mathrm{DA}-\mathrm{D}_{2} \mathrm{R}$ protein expression was increased, with no reported change in the DA- $\mathrm{D}_{1} \mathrm{R}$. Treadmill exercise also resulted in an increase in DA- $\mathrm{D}_{2} \mathrm{R}$ transcript within MSNs of the dorsal striatum supporting the regulatory role of exercise at the level of gene expression $[1,2]$. Using positron emission tomography imaging with $\left[{ }^{18} \mathrm{~F}\right]$-fallypride, a ligand with high specificity for the DA-D 2 , this effect of exercise in MPTP mice was also observed through increased DA-D $2 R$ binding [27]. These reports are consistent with studies that demonstrate an exercise-induced increase of DA$\mathrm{D}_{2} \mathrm{R}$ mRNA, protein, and binding in the striatum of healthy non-dopamine depleted rodents [28-30]. Translating these MPTP animal findings to clinical studies, an exercise-induced increase in $\mathrm{DA}-\mathrm{D}_{2} \mathrm{R}$ expression was also observed in individuals newly diagnosed with PD [31]. After an 8-week regimen of intensive treadmill training, subjects who underwent PET-imaging demonstrated an $80 \%$ increase in binding of $\left[{ }^{18} \mathrm{~F}\right]$ fallypride within the dorsal caudate nucleus compared to pre-exercise baseline values [31]. While the relationship between exercise-induced motor benefits in $\mathrm{PD}$ and increased $\mathrm{DA}-\mathrm{D}_{2} \mathrm{R}$ expression in humans is unknown, studies in healthy animals suggest that striatal DA- $\mathrm{D}_{2} \mathrm{R}$ function and its role in the establishment and maintenance of motor skill learning, may underlie this benefit [32,33]. For example, electrophysiological studies within the striatum of animals, in conjunction with a pharmacologically specific blockade of DA$\mathrm{D}_{2} \mathrm{Rs}$, have shown that antagonism of the DA- $\mathrm{D}_{2} \mathrm{R}$ in either early or late phases of motor skill learning leads to impairment in glutamatergic-dependent synaptic potentiation and motor learning [33]. These studies also demonstrate that $\mathrm{DA}-\mathrm{D}_{2} \mathrm{R}$ related synaptic plasticity that is responsible for motor learning is localized to the dorsal striatum. Further support for the role of DA$\mathrm{D}_{2} \mathrm{R}$ and motor learning comes from studies in rodents by Beeler and colleagues [25, 32]. These researchers demonstrate that the DA- $\mathrm{D}_{2} \mathrm{R}$ is also important in the maintenance of learned motor behaviors since pharmacological blockade of the DA- $\mathrm{D}_{2} \mathrm{R}$, and not the DA- $\mathrm{D}_{1} \mathrm{R}$, in rodents leads to loss of a learned motor skill.

In addition to its role in motor performance, preliminary studies in animals suggest that an exerciseinduced increase in dorsal striatal DA- $\mathrm{D}_{2} \mathrm{R}$ expression may also contribute to the reported exercise related improvements in executive function, including behavioral flexibility [34]. Specifically, studies have shown that 18 days of exercise can improve discrimination testing in a set-shifting, cross-maze task in healthy rodents. This exercise benefit was reversed through selective pharmacological blockade of the DA- $\mathrm{D}_{2} \mathrm{R}$. Taken together animal studies support that exercise induced increase in DA availability along with increased DA- $\mathrm{D}_{2}$ Rexpression in the dorsal striatum and its related cortical circuitry may contribute to exercise related effects in neuroplasticity and behavioral benefits in PD. Future studies in humans are clearly needed to confirm this relationship.

\section{EXERCISE EFFECTS ON GLUTAMATE NEUROTRANSMISSION IN ANIMAL MODELS}

Glutamate neurotransmission is also important in synaptic function and especially in learning and 
memory as demonstrated by its role in mediating both long-term potentiation (LTP) and long-term depression (LTD) [35, 36]. These electrophysiological properties of synaptic connectivity are dictated by specific receptor subtypes, especially the NMDA (N-methyl-D-aspartate) and AMPA (alpha-amino-3hydroxy-5-methyl-4-isoxazolepropionic acid) receptor, not only through long term synapse specific expression, but also by fast trafficking from intracellular stores to sites within the postsynaptic density [36]. Changes in glutamate receptor subtype expression (i.e. neuroplasticity) and localization on neuronal electrophysiological properties are the direct result of experience-dependent events, including exercise. For example, using the MPTP-lesioned mouse model, we have reported exercise-induced changes in synaptic expression of specific receptor subunits of the AMPA receptor [37]. As previously mentioned above, DAdepletion leads to structural and functional changes in striatal MSNs, including the loss of predominantly cortico-striatal synaptic connections (in both direct and indirect projection pathways) and increased glutamatergic drive in remaining cortico-striatal synaptic connections [38]. In the MPTP mouse, exercise is able to reverse this aberrant hyperactive glutamatergic state by two would-be processes. First, exercise alters glutamatergic receptor subunit expression, especially the AMPA receptor subunit GluA2, particularly localized to indirect $\mathrm{DA}-\mathrm{D}_{2} \mathrm{R}$ containing MSNs [37]. On striatal MSNs, exercise increases the relative expression of GluA2, a calcium impermeable AMPA receptor subunit type, from the calcium permeable AMPA receptor GluA1. Electrophysiological correlates demonstrate that exercise reduces synaptic excitability and postexcitatory synaptic potentials [37]. Second, exercise reduces the presynaptic storage of glutamate, as measured through electron microscopy [1]. Taken together, exercise reduces aberrant glutamatergic drive, thus, restoring cortico-striatal circuit function.

\section{EFFECTS OF EXERCISE ON DENDRITIC SPINE DENSITY IN THE BASAL GANGLIA}

In addition to functional synaptic changes, the loss of DA leads to morphological changes in glutamatergic synapses, including a decrease in dendritic spine density and disruption of connectivity in the motor circuit [39]. Dendritic spine loss of MSNs has been reported in post-mortem tissues of patients with $\mathrm{PD}$, as well as in the 6-OHDA and MPTP rodent models of DAdepletion [40-42]. In addition, studies have suggested that dendritic spine loss occurs predominantly on the DA-D ${ }_{2}$ R-containing MSNs early after DA depletion $[43,44]$, but others have shown that spine loss occurs on both DA-D $2 \mathrm{R}$ and DA-D 1 R following prolonged DA-depletion [41]. One possible effect of exercise toward restoration of the circuitry of the basal ganglia may be through changes in spine density. Studies in healthy rodents subjected to environmental enrichment, voluntary wheel running, and forced treadmill running paradigms have demonstrated an increase in dendritic spine density in cerebellar Purkinje, CA3 hippocampal pyramidal, and layer III cortical neurons [45-49]. Studies in MPTP mice have shown that intensive treadmill running can reverse the loss of dendritic spines on striatal MSNs [50]. Besides effects on dendritic spine density, intensive exercise also leads to the restoration of synapses as indicated by the elevated expression of both presynaptic (Synaptophysin) and postsynaptic (PSD-95) proteins.

\section{EXERCISE TYPE, BLOOD FLOW, AND NEUROGENESIS}

Exercise type can be loosely categorized into predominantly skilled or aerobic exercise. Aerobic exercise is a system of conditioning aimed at enhancing circulatory and respiratory efficiency that improves the body's use of oxygen through vigorous, sustained exercise such as running, swimming, or cycling. This is in contrast to skilled exercise, which is a form of goal-oriented movement in which temporal and/or spatial accuracy is important for achieving pre-determined objectives. The important relationship between the type of exercise and nature of neuroplasticity related changes is underscored by prior work suggesting that rats that have undergone unskilled and repetitive exercise (aerobic exercise) have an increase in the density of capillaries in the brain's motor regions, without an increase in synaptic numbers (as measured by dendritic spine density) [51-53]. This is in contrast to rats that have learned new motor skills (skilled exercise) and have a greater number of synapses per neuron, without an increase in the density of capillaries. Recent studies in animal models, including PD, have begun to further elucidate the differential effects of skilled versus aerobic exercise on neuroplasticity associated with alterations in blood flow. These differential effects of skilled versus aerobic exercise are observed at the level of anatomical specificity (circuit and brain region). Specifically, recent work by our laboratory suggests that skilled compared to non-skilled aerobic training 
differentially affects functional activation of the medial prefrontal cortex in parkinsonian rats during walking [54]. Rats with bilateral, striatal 6-OHDA lesions were exposed to forced exercise for 4 weeks, either on a simple running wheel, considered a form of non-skilled aerobic exercise (AE), or on a complex wheel with irregularly spaced rungs, a form of skilled aerobic exercise (SAE). Cerebral perfusion was mapped during horizontal treadmill walking or at rest using $\left[{ }^{14} \mathrm{C}\right]-$ iodoantipyrine autoradiography, one week after the completion of exercise. SAE compared to AE resulted in greater increases in regional cerebral blood flow (rCBF) during walking and at rest in the prefrontal cortex (prelimbic area). Seed correlation analysis during locomotor walking revealed that SAE compared to AE resulted in a much broader functional connectivity of prefrontal cortex with the striatum providing evidence of frontal-striatal neuroplasticity in these circuits through exercise. In addition, there was also evidence for changes in functional connectivity involving the primary and secondary motor cortices, and primary somatosensory cortex. Lastly, prelimbic cortical activation correlated with restoration of motor function in lesioned rats undergoing skilled aerobic exercise more than with non-skilled aerobic exercise. These results show for the first time that SAE compared to $\mathrm{AE}$ results in enhancement of prefrontal cortical mediated control of motor function. We propose that the SAE paradigm likely required greater effort in motor preparatory processing, motor control and set shifting than that required for the $\mathrm{AE}$, all key roles ascribed to prelimbic cortex $[55,56]$. This suggests that the prefrontal cortex and its associated pathways are a central target for experience-dependent neuroplasticity as a result of SAE. It remains to be proven whether such recruitment of prefrontal cortex by SAE will improve performance of the 6-OHDA rat during set-shifting tasks. If proven, this would confirm the notion that motor rehabilitation programs for PD patients should include a relatively high cognitive demand, such that by forcing patients to practice task-switching over a sufficient number of practice trials, they might be able to overcome their inability to generalize learned actions to different environmental contexts $[57,58]$. In addition, future research will need to examine whether any recruitment of prefrontal cortex by SAE is due to changes in dopaminergic pathways. While dopaminergic dysfunction in prefrontal cortex is an early feature of PD and has been linked to dopaminergic loss in the caudate and substantia nigra [59-63], its role in shaping cognitive deficits and responding to an exercise intervention remains to be determined.
In the healthy rodent brain, studies are beginning to demonstrate that the types of exercise and activity can differentially influence the various stages of neurogenesis including cell migration, differentiation, maturation and circuit integration [64, 65]. Neurogenesis is the birth of new neurons. Within the adult mammalian brain there are several unique regions that display the birth of new cells throughout life, including the granular cell layer of the hippocampus, the subventricular zone, and the prefrontal cortex [66]. It is well established that exercise (and environmental enrichment, especially those designs that incorporate running wheels) enhances neurogenesis in the healthy rodent brain [67, 68]. For example, in both young and aged mice, voluntary wheel running has been positively correlated with increased hippocampal neurogenesis and improved memory as demonstrated by enhanced water maze performance [67, 69]. Interestingly, rodents exposed to an enriched environment that incorporates aspects of skilled activity and cognitive engagement compared to rodents exposed to voluntary running wheel show greater cognitive flexibility in the Morris Water Maze. This improvement may be due to both neurogenesis and enhanced neuronal incorporation into hippocampal circuitry [70]. Such studies suggest that while many different types of physical activity promote the survival of these newborn cells, migration, and integration may be dependent on the degree of cognitive (skilled) engagement [71, 72]. The effect of skilled exercise on stages of neurogenesis may be due to its influence on the proliferation of astrocytes, activation of microglia, and expression of factors, such as neurotrophic factors and its receptors, which are known to be important in regulating neuroplasticity and synaptogenesis in brain regions where the birth of new cells are promoted [73-75].

In rodent models of neurological disorders where reduced neurogenesis is evident, including mouse models of Alzheimer's disease, exercise has been shown to elevate hippocampal neurogenesis and delay deficits in learning and memory [76]. However, some studies have shown elevated or no effects of exercise in neurogenesis in the context of disease, such as Huntington's disease [77-79]. In animal models of PD, exercise may facilitate neurogenesis in the hippocampus and subventricular zone, similar to reports with wild-type animals, however, there are few reports showing enhanced neurogenesis within the damaged striatum or midbrain regions with physical activity $[80,81]$. Clearly a major gap in our knowledge is whether exercise or the type of exercise influences different stages of neurogenesis in brain regions affected by disease. 


\section{EXERCISE AND THE COUPLING OF NEURONAL ACTIVATION AND CEREBRAL BLOOD FLOW}

As described in previous sections, skilled exercise may lead to the recruitment and activation of neurons in specific circuits within the brain. On the other hand, aerobic exercise may have more global effects on the entire brain including lowering the threshold for neuroplasticity to occur through the expression of neurotrophic factors or other modulators of synaptic plasticity as well as increasing $\mathrm{rCBF}$ [82]. However, the activation of neurons through engagement in skilled exercise and the modulation of blood flow through aerobic exercise may not be mutually exclusive processes. Rather they may promote and regulate neuroplasticity through overlapping and integrated mechanisms. One potential scenario may be that intensive skilled exercise with a resultant increase in neuronal activity to a specific circuit (a motor circuit for example) may result in elevated demand for regional oxygen consumption (resulting in oxygen depletion within this region). Elevated oxygen consumption in turn, can activate a number of regulatory signals that respond to changes in metabolic expenditure. For example, the hypoxia-inducible transcription factor 1 alpha (HIF1alpha) is activated under conditions of low tissue oxygenation, the result of increased metabolic demand [83]. Acute, or moderate to intensive aerobic exercise has been shown to induce transient cerebral hypoxia, which is largely sensed by HIF-1alpha [84]. Important to neuroplasticity, HIF-1alpha regulates the expression of a wide array of downstream target genes implicated in promoting neurogenesis, synaptogenesis, and angiogenesis [85]. Furthermore, HIF-1alpha modulates the expression of genes essential for increasing fuel availability, such as glucose transporters (GLUT-1 and GLUT-3) and enzymes that participate in the glycolytic pathway. These enzymes in turn may facilitate and support synaptic strength and connectivity [84]. Thus, exercise, through orchestrating the recruitment of circuitry, high neuronal activity along with increasing cellular metabolic energy demand, leads to the activation of a cascade of genes important for neuroplasticity, repair, and the establishment of homeostasis. These mechanism linking exercise and neuroplasticity may also involve increased $\mathrm{rCBF}$ to activated brain regions leading to a number of important consequences including: (i) increasing the availability of biomolecules responding to increased energy demand, (ii) removal of waste materials and maintenance of cellular homeostasis, (iii) increased delivery of neurotrophic factors such as BDNF, (iv) altering the blood brain barrier to allow the targeted passage of biomolecules and circulating cells such as macrophages to activated sites, and (v) delivery of biomolecules involved in the formation of synaptic connections. Thus, exercise may incorporate either or both mechanisms to facilitate neuroplasticity. A major gap in knowledge is the precise cause-effect relationship between elevated metabolic demand and altered CBF. Metabolically high demand neuronal circuits can release nitric oxide synthase (NOS) and angiogenic factors to increase blood flow to sites where there is demand [86-88]. On the other hand, metabolically active neuronal circuits may reinforce regional increases in $\mathrm{CBF}$.

\section{CONCLUSIONS}

Animal studies have been instrumental in providing evidence for exercise's role in neuroplasticity of corticostriatal circuits that are profoundly affected in PD. This evidence includes exercise's role in modulating DA and glutamate neurotransmission, synaptogenesis and increased regional cerebral blood flow. In addition, recent evidence supports that the type of exercise may have regional effects on brain circuitry, with skilled exercise differentially affecting frontal related circuits more so than pure aerobic exercise. Although clearly more research is needed to address major gaps in our knowledge, we hypothesize that skilled compared to aerobic exercise has different effects on neuroplasticity, but that these effects may not be mutually exclusive. For example, the potential effects of different types of exercise on inducing neuroplasticity in a circuit specific manner may occur through synergistic mechanisms that include the coupling of an increasing neuronal metabolic demand with a corresponding increase in regional blood flow. Thus, both types of exercise may be important for facilitating neuroplasticity. In Fig. 2 we illustrate that most exercises lie within a spectrum between aerobic and skilled exercise. For example, peddling on a recumbent bicycle may be considered predominantly aerobic with minimal skill or cognitive engagement. On the other end of the spectrum juggling may represent a highly skilled task with minimal aerobic involvement. However, many exercises such as swimming and running involve a combination of both skilled and aerobic exercise. Elucidation of the relative contribution of different types of exercise on neuroplasticity and motor and cognitive improvement in PD may provide mechanistic insights important to facilitate brain repair and modify disease progression. 


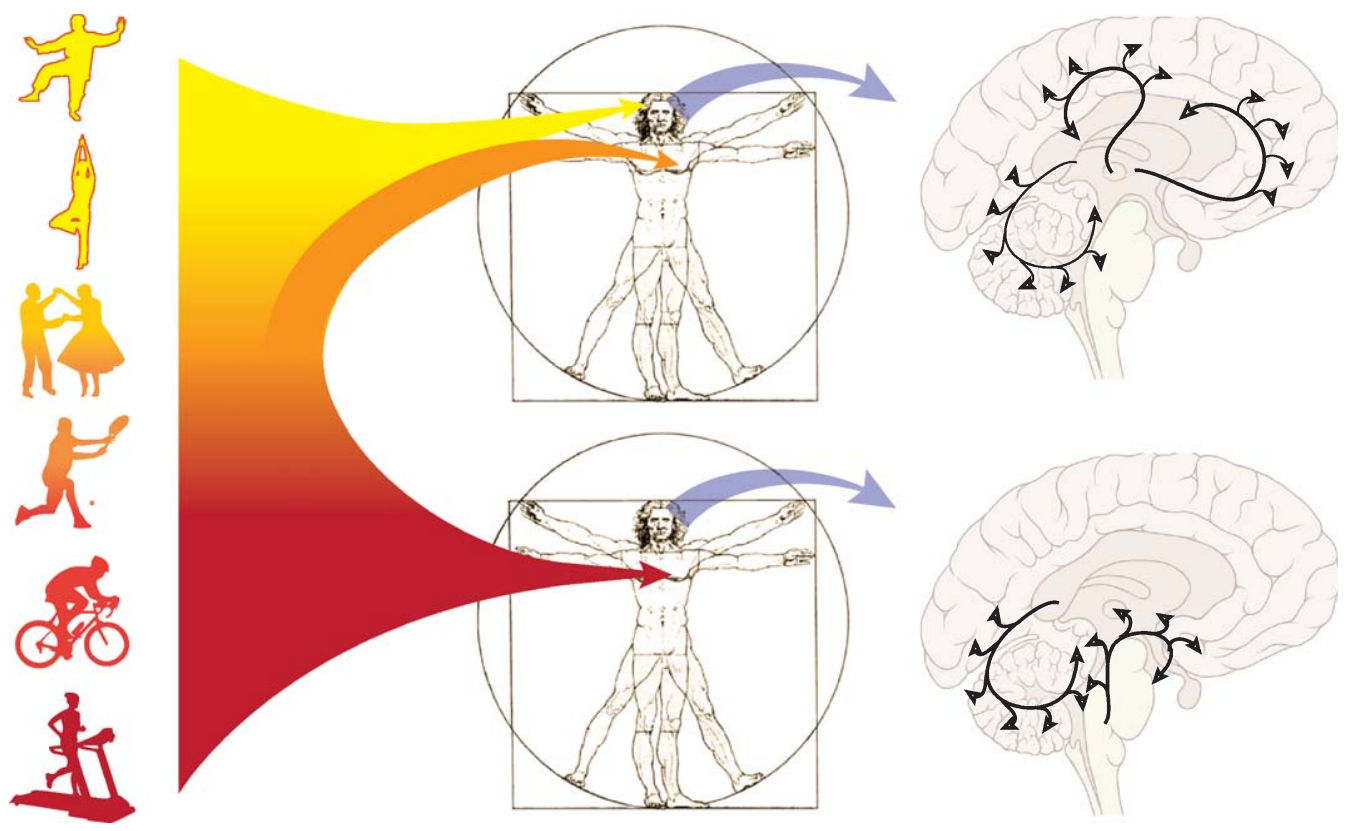

Fig. 2. Physical activity spans the spectrum from aerobic to skilled exercise. Recent exercise studies in animal models of PD are beginning to support the differential effects of aerobic versus skilled exercise on the establishment and maintenance of brain circuitry. In this Figure we illustrate these concepts. One potential hypothesis highlights aerobic exercise that may lead to a broad increase in cerebral blood flow, including within those brain circuits in the basal ganglia and cerebellum involved in motor control. Other global factors may also be activated including reduced oxidative stress, reduced neuro-inflammation, and increased expression of neurotrophic factors. This is in contrast to skilled exercise that entails perceptual and a higher level cognitive processing that may specifically target prefrontal and associated cortical circuits important for executive function.

\section{ACKNOWLEDGMENTS}

The authors would like to acknowledge the support of the NINDS R01 NS44327, NICHD R01 HD060630, U.S. Army NETRP (Grant \# W81XWH-04-1-0444), CTSI/CTSA of USC, and Zumberge Foundation of USC. This work would not be possible without the generous support of the Don Roberto Gonzales family Foundation and their interest in PD research and the importance of exercise/healthy lifestyle for patients and families. A special thanks to Friends of the USC Parkinson's Disease Research Group including George and Mary Lou Boone, Walter and Susan Doniger. Special thank you to Lauren Hawthorne for the design of the figures. This paper is dedicated to our colleague Dr. Garnik Akopian.

\section{CONFLICT OF INTEREST}

The authors state that there is no financial conflict of interest regarding the studies discussed in this manuscript.

\section{REFERENCES}

[1] Fisher BE, Petzinger GM, Nixon K, Hogg E, Bremmer $\mathrm{S}$, Meshul CK, et al. Exercise-induced behavioral recovery and neuroplasticity in the 1-methyl-4-phenyl-1,2,3,6tetrahydropyridine-lesioned mouse basal ganglia. J Neurosci Res. 2004;77(3):378-90.

[2] Petzinger GM, Walsh JP, Akopian G, Hogg E, Abernathy A, Arevalo $\mathrm{P}$, et al. Effects of treadmill exercise on dopaminergic transmission in the 1-methyl-4-phenyl-1,2,3,6tetrahydropyridine-lesioned mouse model of basal ganglia injury. J Neurosci. 2007;27(20):5291-300.

[3] Real CC, Ferreira AF, Hernandes MS, Britto LR, Pires RS. Exercise-induced plasticity of AMPA-type glutamate receptor subunits in the rat brain. Brain Res. 2010;1363:63-71.

[4] VanLeeuwen JE, Petzinger GM, Walsh JP, Akopian GK, Vuckovic M, Jakowec MW. Altered AMPA receptor expression with treadmill exercise in the 1-methyl-4-phenyl1,2,3,6-tetrahydropyridine-lesioned mouse model of basal ganglia injury. J Neurosci Res. 2010;88(3):650-68.

[5] Blomstrand E, Perrett D, Parry-Billings M, Newsholme EA Effect of sustained exercise on plasma amino acid concentrations and on 5-hydroxytryptamine metabolism in six different brain regions in the rat. Acta Physiol Scand. 1989;136(3): 473-81.

[6] Brown BS, Payne T, Kim C, Moore G, Krebs P, Martin W. Chronic response of rat brain norepinephrine and serotonin levels to endurance training. J Appl Physiol. 1979;46(1): 19-23. 
[7] Semenova TP, Ivanov VA, Tret'yak TM. Brain levels of noradrenalin, dopamine, and serotonin in rats with different levels of motor activity. Neurosci Behav Physiol. 1981;11(2): 153-5.

[8] Pagliari R, Peyrin L. Norepinephrine release in the rat frontal cortex under treadmill exercise: A study with microdialysis. J Appl Physiol. 1995;78(6):2121-30.

[9] Uchida S, Suzuki A, Kagitani F, Hotta H. Responses of acetylcholine release and regional blood flow in the hippocampus during walking in aged rats. J Physiol Sci. 2006;56(3): 253-7.

[10] Nakajima K, Uchida S, Suzuki A, Hotta H, Aikawa Y. The effect of walking on regional blood flow and acetylcholine in the hippocampus in conscious rats. Auton Neurosci. 2003;103(1-2):83-92.

[11] Kreitzer AC, Malenka RC. Striatal Plasticity and Basal Ganglia Circuit Function. Neuron. 2008;60(4):543-54

[12] Leh SE, Petrides M, Strafella AP. The neural circuitry of executive functions in healthy subjects and Parkinson's disease. Neuropsychopharmacology. 2010;35(1):70-85.

[13] Dirnberger G, Jahanshahi M. Executive dysfunction in Parkinson's disease: A review. J Neuropsychol. 2013;7(2): 193-224.

[14] Engeln M. Throwing some light on executive function in Parkinson's disease. Mov Disord. 2013;28(8):1052.

[15] Filoteo JV, Reed JD, Litvan I, Harrington DL. Volumetric correlates of cognitive functioning in nondemented patients with Parkinson's disease. Mov Disord. 2014;29(3):360-7.

[16] Floresco SB. Prefrontal dopamine and behavioral flexibility: Shifting from an "inverted-U" toward a family of functions. Front Neurosci. 2013;7:62

[17] Courtiere A, Hardouin J, Burle B, Vidal F, Turle-Lorenzo $\mathrm{N}$, Amalric $\mathrm{M}$, et al. Dynamics of executive control and motor deficits in parkinsonian rats. J Neurosci. 2011;31(33): 11929-33.

[18] Yawata S, Yamaguchi T, Danjo T, Hikida T, Nakanishi S. Pathway-specific control of reward learning and its flexibility via selective dopamine receptors in the nucleus accumbens. Proc Natl Acad Sci U S A. 2012;109(31):12764-9.

[19] Thacker EL, Chen H, Patel AV, McCullough ML, Calle EE, Thun MJ, et al. Recreational physical activity and risk of Parkinson's disease. Mov Disord. 2008;23(1):69-74.

[20] Pitkala KH, Poysti MM, Laakkonen ML, Tilvis RS, Savikko $\mathrm{N}$, Kautiainen $\mathrm{H}$, et al. Effects of the Finnish Alzheimer Disease Exercise Trial (FINALEX): A randomized controlled trial. JAMA Internal Medicine. 2013;173(10):894-901.

[21] Voelcker-Rehage C, Godde B, Staudinger UM. Cardiovascular and coordination training differentially improve cognitive performance and neural processing in older adults. Front Hum Neurosci. 2011;5:26

[22] Chen H, Zhang SM, Schwarzschild MA, Hernan MA, Ascherio A. Physical activity and the risk of Parkinson disease. Neurology. 2005;64(4):664-9.

[23] Jakowec MW, Petzinger GM. 1-methyl-4-phenyl-1,2,3,6tetrahydropyridine-lesioned model of parkinson's disease, with emphasis on mice and nonhuman primates. Comp Med. 2004;54(5):497-513.

[24] Schwarting RK, Huston JP. The unilateral 6hydroxydopamine lesion model in behavioral brain research. Analysis of functional deficits, recovery and treatments. Prog Neurobiol. 1996;20(2-3):275-331.

[25] Beeler JA, Cao ZF, Kheirbek MA, Ding Y, Koranda J, Murakami M, et al. Dopamine-dependent motor learning: Insight into levodopa's long-duration response. Ann Neurol. 2010;67(5):639-47.
[26] Beaulieu JM, Gainetdinov RR. The Physiology, Signaling, and Pharmacology of Dopamine Receptors. Pharmacol Rev. 2011;63(1):182-217.

[27] Vuckovic MG, Li Q, Fisher B, Nacca A, Leahy RM, Walsh $\mathrm{JP}$, et al. Exercise elevates dopamine D2 receptor in a mouse model of Parkinson's disease: In vivo imaging with [(1)F]fallypride. Mov Disord. 2010;25(16):2777-84.

[28] Foley TE, Fleshner M. Neuroplasticity of Dopamine Circuits After Exercise: Implications for central fatigue. Neuromolecular Med. 2008;10(2):67-80.

[29] Gilliam PE, Spirduso WW, Martin TP, Walters TJ, Wilcox RE, Farrar RP. The effects of exercise training on $[3 \mathrm{H}]-$ spiperone binding in rat striatum. Pharmacol Biochem Behav. 1984;20(6):863-7.

[30] MacRae PG, Spirduso WW, Cartee GD, Farrar RP, Wilcox RE. Endurance training effects on striatal D2 dopamine receptor binding and striatal dopamine metabolite levels. Neurosci Lett. 1987;79(1-2):138-44.

[31] Fisher BE, Li Q, Nacca A, Salem GJ, Song J, Yip J, et al. Treadmill exercise elevates striatal dopamine D2 receptor binding potential in patients with early Parkinson's disease. Neuroreport. 2013;24(10):509-14.

[32] Beeler JA, Frank MJ, McDaid J, Alexander E, Turkson S, Bernandez MS, et al. A role for dopamine-mediated learning in the pathophysiology and treatment of Parkinson's disease. Cell Rep. 2012;2(6):1747-61.

[33] Yim AJ, Andersen ML, Soeiro AC, Tufik S, Oliveira MG. Acute systemic blockade of D2 receptors does not accelerate the extinction of cocaine-associated place preference. Brain Res. 2009; 1304:122-8.

[34] Eddy MC, Stansfield KJ, Green JT. Voluntary exercise improves performance of a discrimination task through effects on the striatal dopamine system. Learn Mem. 2014;21(7): 334-7.

[35] Bliss TV, Cooke SF. Long-term potentiation and longterm depression: A clinical perspective. Clinics (Sao Paulo). 2011;66 Suppl 1:3-17.

[36] Huganir RL, Nicoll RA. AMPARs and Synaptic Plasticity: The last 25 years. Neuron. 2013;80(3):704-17.

[37] Kintz N, Petzinger GM, Akopian G, Ptasnik S, Williams C, Jakowec MW, et al. Exercise modifies alpha-amino-3hydroxy-5-methyl-4-isoxazolepropionic acid receptor expression in striatopallidal neurons in the 1-methyl-4-phenyl1,2,3,6-tetrahydropyridine-lesioned mouse. J Neurosci Res. 2013;91(11):1492-507

[38] Calabresi P, Mercuri NB, Sancesario G, Bernardi G. Electrophysiology of dopamine-denervated striatal neurons. Implications for Parkinson's disease. Brain. 1993;116(Pt 2): 433-52.

[39] Elsworth JD, Leranth C, Redmond DE, Roth RH. Loss of asymmetric spine synapses in prefrontal cortex of motor-asymptomatic, dopamine-depleted, cognitively impaired MPTP-treated monkeys. Int J Neuropsychopharmacol. 2012:1-8

[40] Smith Y, Villalba RM, Raju DV. Striatal spine plasticity in Parkinson's disease: Pathological or not? Parkinsonism Relat Disord. 2009;15 Suppl 3:S156-61.

[41] Villalba RM, Lee H, Smith Y. Dopaminergic denervation and spine loss in the striatum of MPTP-treated monkeys. Exp Neurol. 2009;215(2):220-7.

[42] Pickel VM, Johnson E, Carson M, Chan J. Ultrastructure of spared dopamine terminals in caudate-putamen nuclei of adult rats neonatally treated with intranigral 6hydroxydopamine. Brain Res Dev Brain Res. 1992;70(1): $75-86$ 
[43] Cazorla M, Shegda M, Ramesh B, Harrison NL, Kellendonk C. Striatal D2 receptors regulate dendritic morphology of medium spiny neurons via Kir2 channels. J Neurosci. 2012;32(7):2398-409.

[44] Fasano C, Bourque MJ, Lapointe G, Leo D, Thibault D, Haber $\mathrm{M}$, et al. Dopamine facilitates dendritic spine formation by cultured striatal medium spiny neurons through both D1 and D2 dopamine receptors. Neuropharmacology. 2013;67:43243.

[45] Eadie BD, Redila VA, Christie BR. Voluntary exercise alters the cytoarchitecture of the adult dentate gyrus by increasing cellular proliferation, dendritic complexity, and spine density. J Comp Neurol. 2005;486(1):39-47.

[46] Nelson AJ, Iwamoto GA. Reversibility of exercise-induced dendritic attenuation in brain cardiorespiratory and locomotor areas following exercise detraining. J Appl Physiol. 2006;101(4):1243-51.

[47] Hu XL, Bergstrom SA, Brink M, Ronnback A, Dahlqvist P. Enriched environment increases spinophilin mRNA expression and spinophilin immunoreactive dendritic spines in hippocampus and cortex. Neurosci Lett. 2010;476(2):79-83.

[48] Kozorovitskiy Y, Gross CG, Kopil C, Battaglia L, McBreen M, Stranahan AM, et al. Experience induces structural and biochemical changes in the adult primate brain. Proc Natl Acad Sci U S A. 2005;102(48):17478-82

[49] Leggio MG, Mandolesi L, Federico F, Spirito F, Ricci B, Gelfo F, et al. Environmental enrichment promotes improved spatial abilities and enhanced dendritic growth in the rat. Behav Brain Res. 2005;163(1):78-90

[50] Toy WA, Petzinger GM, Leyshon BJ, Akopian GK, Walsh JP, Hoffman MV, et al. Treadmill exercise reverses dendritic spine loss in direct and indirect striatal medium spiny neurons in the 1-methyl-4-phenyl-1,2,3,6-tetrahydropyridine (MPTP) mouse model of Parkinson's disease. Neurobiol Dis. 2014;63:201-9.

[51] Black JE, Isaacs KR, Anderson BJ, Alcantara AA, Greenough WT. Learning causes synaptogenesis, whereas motor activity causes angiogenesis, in cerebellar cortex of adult rats. Proc Natl Acad Sci U S A. 1990;87(14):5568-72.

[52] Garcia PC, Real CC, Ferreira AF, Alouche SR, Britto LR, Pires RS. Different protocols of physical exercise produce different effects on synaptic and structural proteins in motor areas of the rat brain. Brain Res. 2012;1456:36-48.

[53] Kleim JA, Barbay S, Cooper NR, Hogg TM, Reidel CN, Remple MS, et al. Motor learning-dependent synaptogenesis is localized to functionally reorganized motor cortex. Neurobiol Learn Mem. 2002;77(1):63-77.

[54] Wang Z, Guo Y, Myers KG, Heintz R, Peng YH, Maarek $\mathrm{JM}$, et al. Exercise alters resting-state functional connectivity of motor circuits in parkinsonian rats. Neurobiol Aging. 2015;36(1):536-44.

[55] Rosano C, Studenski SA, Aizenstein HJ, Boudreau RM, Longstreth WT Jr., Newman AB. Slower gait, slower information processing and smaller prefrontal area in older adults. Age Ageing. 2012;41(1):58-64.

[56] Risterucci C, Terramorsi D, Nieoullon A, Amalric M. Excitotoxic lesions of the prelimbic-infralimbic areas of the rodent prefrontal cortex disrupt motor preparatory processes. Eur J Neurosci. 2003;17(7):1498-508.

[57] Onla-or S, Winstein CJ. Determining the optimal challenge point for motor skill learning in adults with moderately severe Parkinson's disease. Neurorehabil Neural Repair. 2008;22(4):385-95.
[58] Petzinger GM, Fisher BE, McEwen S, Beeler JA, Walsh JP, Jakowec MW. Exercise-enhanced neuroplasticity targeting motor and cognitive circuitry in Parkinson's disease. Lancet Neurol. 2013;12(7):716-26.

[59] Berti V, Polito C, Ramat S, Vanzi E, De Cristofaro MT, Pellicano G, et al. Brain metabolic correlates of dopaminergic degeneration in de novo idiopathic Parkinson's disease. Eur J Nucl Med Mol Imaging. 2010;37(3):537-44.

[60] Carbon M, Eidelberg D. Functional imaging of sequence learning in Parkinson's disease. J Neurol Sci. 2006;248(12):72-7.

[61] Kaasinen V, Nurmi E, Bruck A, Eskola O, Bergman J, Solin $\mathrm{O}$, et al. Increased frontal [(18)F]fluorodopa uptake in early Parkinson's disease: Sex differences in the prefrontal cortex. Brain. 2001;124(Pt 6):1125-30.

[62] Polito C, Berti V, Ramat S, Vanzi E, De Cristofaro MT, Pellicano $\mathrm{G}$, et al. Interaction of caudate dopamine depletion and brain metabolic changes with cognitive dysfunction in early Parkinson's disease. Neurobiol Aging. 2012;33(1):206 e29-39.

[63] Sawamoto N, Piccini P, Hotton G, Pavese N, Thielemans K Brooks DJ. Cognitive deficits and striato-frontal dopamine release in Parkinson's disease. Brain. 2008;131(Pt 5):1294302.

[64] Clark PJ, Bhattacharya TK, Miller DS, Kohman RA, Deyoung EK, Rhodes JS. New neurons generated from running are broadly recruited into neuronal activation associated with three different hippocampus-involved tasks. Hippocampus. 2012;22(9):1860-7.

[65] Klaus F, Hauser T, Slomianka L, Lipp HP, Amrein I. A reward increases running-wheel performance without changing cell proliferation, neuronal differentiation or cell death in the dentate gyrus of C57BL/6 mice. Behav Brain Res. 2009;204(1):175-81.

[66] Lie DC, Song H, Colamarino SA, Ming GL, Gage FH. Neurogenesis in the adult brain: New strategies for central nervous system diseases. Annu Rev Pharmacol Toxicol. 2004;44:399421.

[67] van Praag H, Kempermann G, Gage FH. Running increases cell proliferation and neurogenesis in the adult mouse dentate gyrus. Nat Neurosci. 1999;2(3):266-70.

[68] Opendak M, Gould E. Adult neurogenesis: A substrate for experience-dependent change. Trends Cogn Sci. 2015.

[69] Kempermann G, Gast D, Gage FH. Neuroplasticity in old age: Sustained fivefold induction of hippocampal neurogenesis by long-term environmental enrichment. Ann Neurol. 2002;52(2):135-43.

[70] Garthe A, Roeder I, Kempermann G. Mice in an enriched environment learn more flexibly because of adult hippocampal neurogenesis. Hippocampus. 2015. Epub, in Press. doi: 10.10 02/hipo. 22520

[71] Curlik DM, 2nd, Shors TJ. Training your brain: Do mental and physical (MAP) training enhance cognition through the process of neurogenesis in the hippocampus? Neuropharmacology. 2012;64:506-14.

[72] Bednarczyk MR, Hacker LC, Fortin-Nunez S, Aumont A, Bergeron R, Fernandes KJ. Distinct stages of adult hippocampal neurogenesis are regulated by running and the running environment. Hippocampus. 2011;21(12):1334-47.

[73] Ehninger D, Kempermann G. Regional effects of wheel running and environmental enrichment on cell genesis and microglia proliferation in the adult murine neocortex. Cereb Cortex. 2003;13(8):845-51. 
[74] Kohman RA, DeYoung EK, Bhattacharya TK, Peterson LN, Rhodes JS. Wheel running attenuates microglia proliferation and increases expression of a proneurogenic phenotype in the hippocampus of aged mice. Brain Behav Immun 2012;26(5):803-10.

[75] Bernardi C, Tramontina AC, Nardin P, Biasibetti R, Costa AP, Vizueti AF, et al. Treadmill exercise induces hippocampal astroglial alterations in rats. Neural Plast. 2013;2013:709732.

[76] Kim YM, Ji ES, Kim SH, Kim TW, Ko IG, Jin JJ, et al. Treadmill exercise improves short-term memory by enhancing hippocampal cell proliferation in quinolinic acid-induced Huntington's disease rats. Journal of Exercise Rehabilitation. 2015;11(1):5-11.

[77] Catlow BJ, Rowe AR, Clearwater CR, Mamcarz M, Arendash GW, Sanchez-Ramos J. Effects of environmental enrichment and physical activity on neurogenesis in transgenic PS1/APP mice. Brain Res. 2009;1256:173-9.

[78] Kohl Z, Kandasamy M, Winner B, Aigner R, Gross C, Couillard-Despres S, et al. Physical activity fails to rescue hippocampal neurogenesis deficits in the R6/2 mouse model of Huntington's disease. Brain Res. 2007;1155:24-33.

[79] Potter M, Yuan C, Ottenritter C, Mughal M, van Praag $\mathrm{H}$. Exercise is not beneficial and may accelerate symptom onset in a mouse model of Huntington's disease. PLoS Curr. 2010;2:RRN1201.

[80] Klaissle P, Lesemann A, Huehnchen P, Hermann A, Storch A, Steiner B. Physical activity and environmental enrichment regulate the generation of neural precursors in the adult mouse substantia nigra in a dopamine-dependent manner. BMC Neurosci. 2012;13(1):132.
[81] Steiner B, Winter C, Hosman K, Siebert E, Kempermann G, Petrus DS, et al. Enriched environment induces cellular plasticity in the adult substantia nigra and improves motor behavior function in the 6-OHDA rat model of Parkinson's disease. Exp Neurol. 2006;199:291-300.

[82] Cotman CW, Berchtold NC, Christie LA. Exercise builds brain health: Key roles of growth factor cascades and inflammation. Trends Neurosci. 2007;30(9):464-72.

[83] Correia SC, Moreira PI. Hypoxia-inducible factor 1: A new hope to counteract neurodegeneration? J Neurochem. 2010;112(1):1-12.

[84] Kinni H, Guo M, Ding JY, Konakondla S, Dornbos D, 3rd, Tran R, et al. Cerebral metabolism after forced or voluntary physical exercise. Brain Res. 2011;1388:48-55.

[85] Sharp FR, Ran R, Lu A, Tang Y, Strauss KI, Glass T, et al. Hypoxic preconditioning protects against ischemic brain injury. NeuroRx. 2004;1(1):26-35.

[86] Toda N, Ayajiki K, Okamura T. Cerebral blood flow regulation by nitric oxide: Recent advances. Pharmacol Rev. 2009;61(1):62-97.

[87] Viboolvorakul S, Patumraj S. Exercise training could improve age-related changes in cerebral blood flow and capillary vascularity through the upregulation of VEGF and eNOS. BioMed Research International. 2014;2014:230791.

[88] Fabel K, Tam B, Kaufer D, Baiker A, Simmons N, Kuo CJ, et al. VEGF is necessary for exercise-induced adult hippocampal neurogenesis. Eur J Neurosci. 2003;18(10):2803-12. 